\title{
EDQM-HC Closure Terminology
}

National Cancer Institute

\section{Source}

National Cancer Institute. EDQM-HC Closure Terminology. NCI Thesaurus. Code

C150108.

Terminology subset about items used to close a container for the purpose of the correct storage and (where appropriate) use of the product. 